Supporting Information

\title{
Pd@Pt Core-shell Concave Decahedra: A Class of Catalysts for the Oxygen Reduction Reaction with Enhanced Activity and Durability
}

Xue Wang, ${ }^{\dagger, \#}$ Madeline Vara, ${ }^{\ddagger}$ Ming Luo, ${ }^{\dagger}$ Hongwen Huang, ${ }^{\dagger}$ Aleksey Ruditskiy, ${ }^{\ddagger}$ Jinho Park, ${ }^{\ddagger}$ Shixiong Bao, ${ }^{\dagger, \#}$ Jingyue Liu, ${ }^{\S}$ Jane Howe, ${ }^{\|}$Miaofang Chi, ${ }^{\perp}$ Zhaoxiong Xie, ${ }^{\#}$ and Younan Xia* ${ }^{*,, \neq}$

The Wallace H. Coulter Department of Biomedical Engineering, Georgia Institute of Technology and Emory University, Atlanta, Georgia 30332, United States

${ }^{\text {\#}}$ State Key Laboratory of Physical Chemistry of Solid Surfaces, Collaborative Innovation Center of Chemistry for Energy Materials, and Department of Chemistry, Xiamen University, Xiamen, Fujian 361005, P. R. China

School of Chemistry and Biochemistry, Georgia Institute of Technology, Atlanta, Georgia 30332, United States

${ }^{\S}$ Department of Physics, Arizona State University, Tempe, Arizona 85287, United States

"Hitachi High-Technologies Canada, Toronto, Ontario M9W6A4, Canada

${ }^{\perp}$ Center for Nanophase Materials Sciences, Oak Ridge National Laboratory, Oak Ridge, Tennessee 37831, United States

*Correspondence to: younan.xia@bme.gatech.edu 

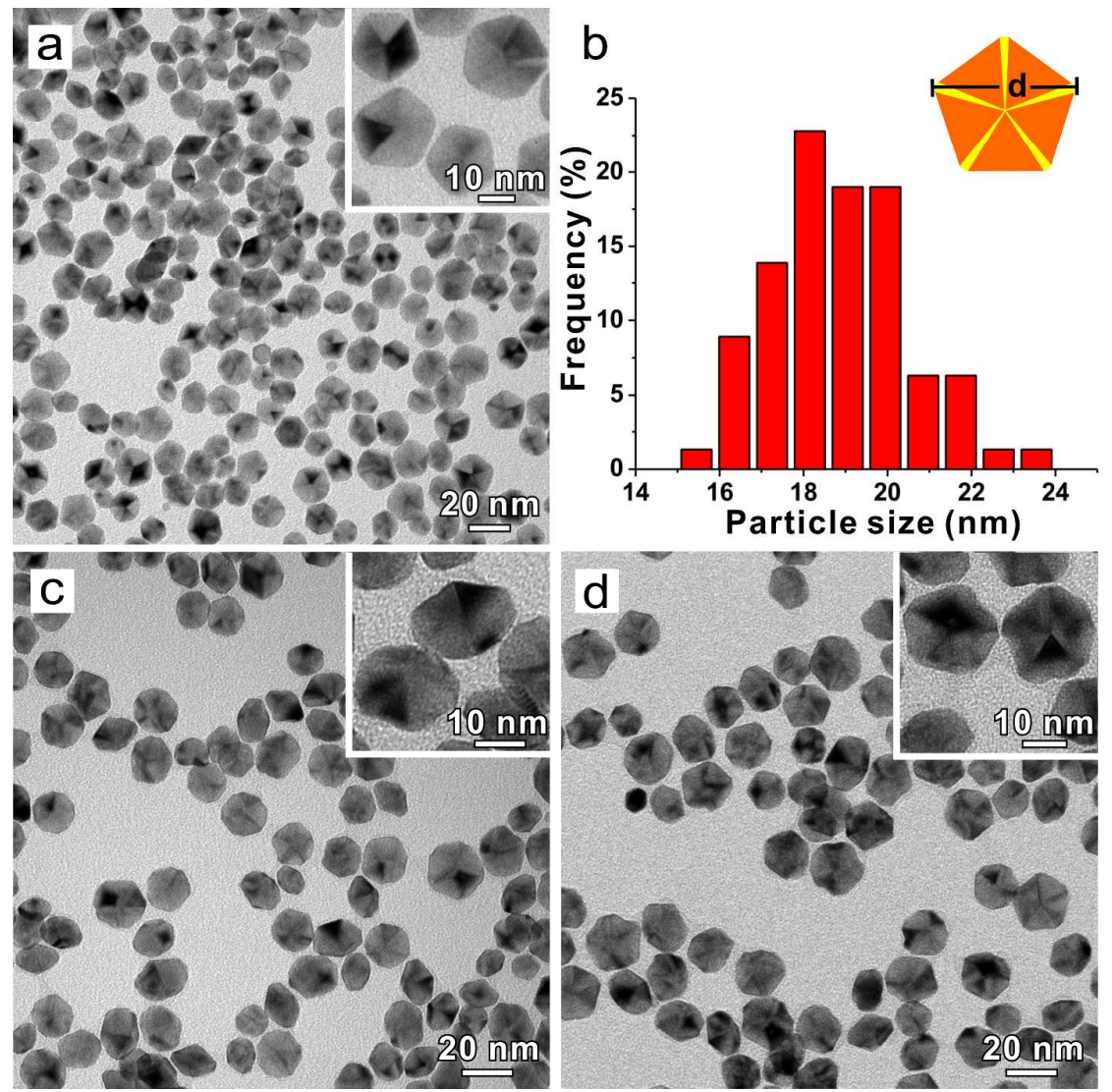

Figure S1. (a) TEM image and (b) size distribution of the Pd decahedral seeds. The size of a decahedron is defined in the inset of (b). (c, d) TEM images of the Pd@Pt concave decahedra with (c) $29.6 \mathrm{wt} \% \mathrm{Pt}$ and (d) $47.5 \mathrm{wt} \% \mathrm{Pt}$, respectively. 


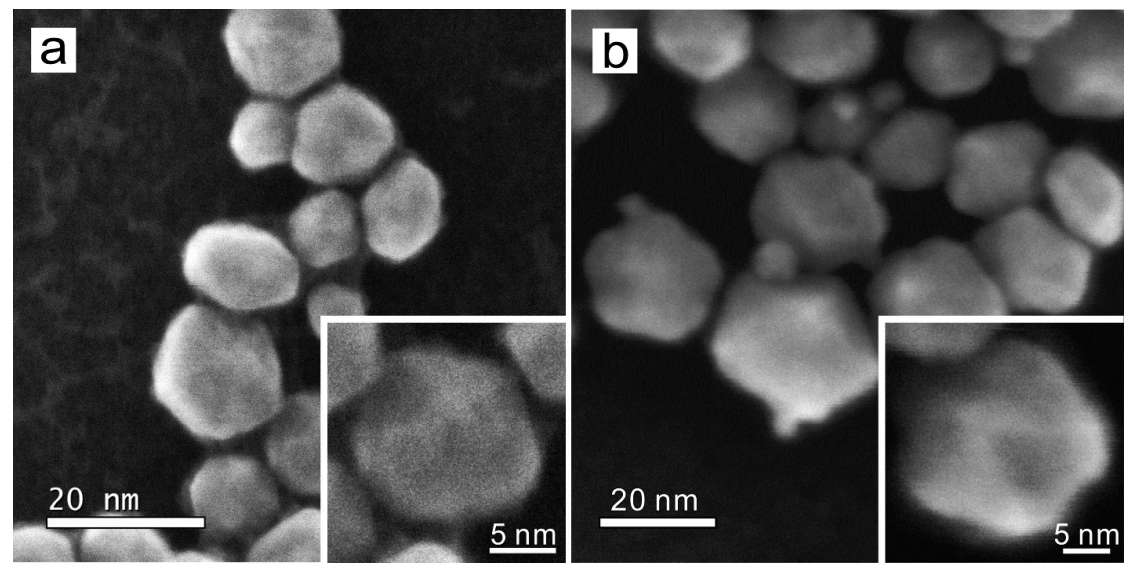

Figure S2. SEM images of the Pd@Pt concave decahedra with (a) 29.6 wt $\%$ Pt and (b) 47.5 $\mathrm{wt} \% \mathrm{Pt}$, respectively. The higher-magnification SEM images in the insets highlight a concave structure on the surface of a particle from each sample. 


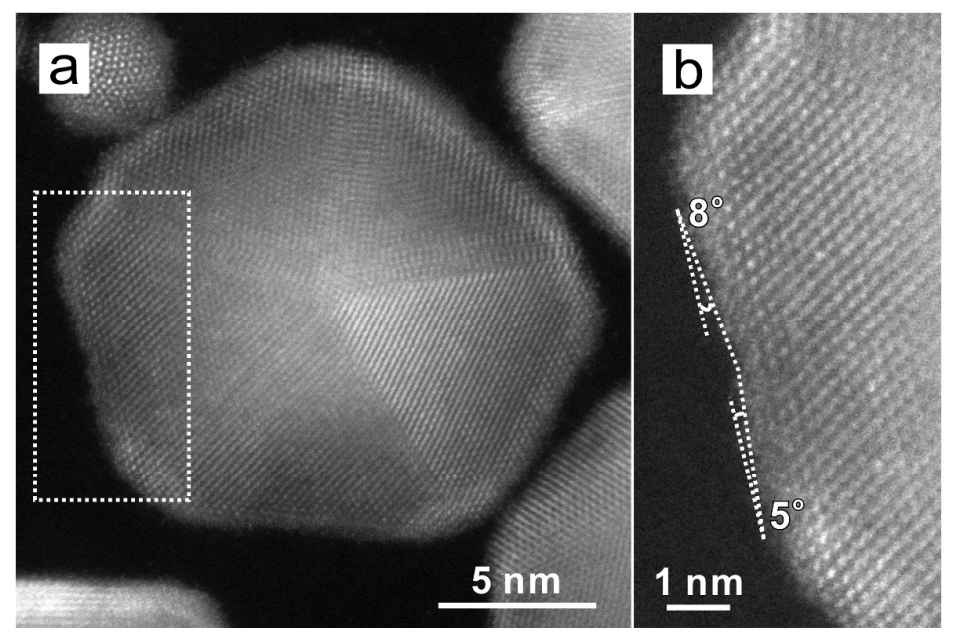

Figure S3. (a) Atomic-resolution HAADF-STEM image of a Pd@Pt core-shell concave decahedron with $29.6 \mathrm{wt} \% \mathrm{Pt}$ viewed along its 5-fold axis (the same particle as imaged in Figure 1b). (b) Atomic-resolution HAADF-STEM image at a higher magnification, which was taken from the left side of the decahedron as marked by a box in (a). The angles between the facets on the edge and the $\{100\}$ facet are $8^{\circ}$ and $5^{\circ}$, which could be further used to identify the high-index facets on the edge, as summarized in Table S1. However, it is very difficult (or impossible) to identify the high-index facets on the faces as the geometry of the decahedron interferes with our ability to obtain a vertical projection of the high-index facet. 


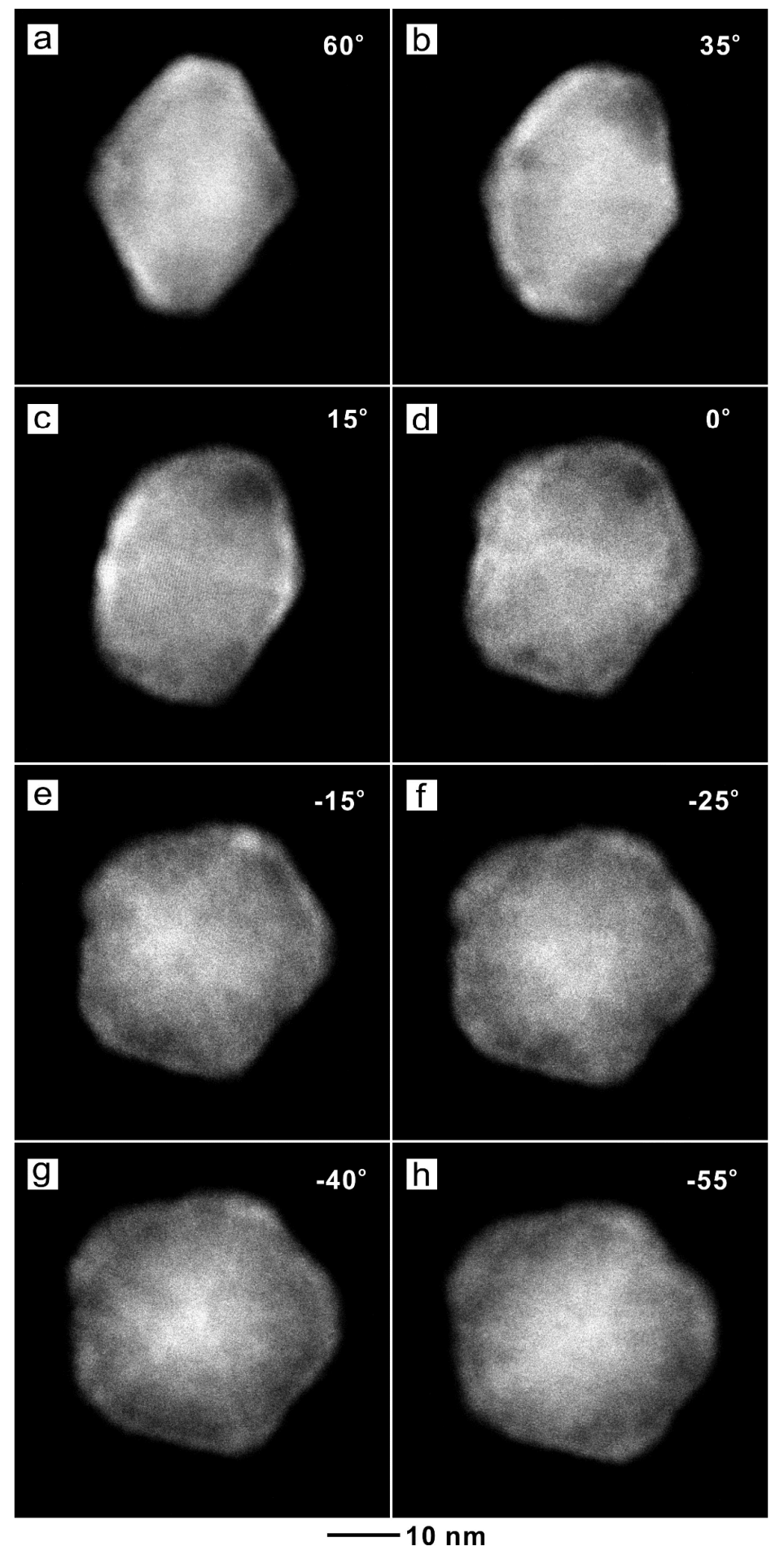

Figure S4. HAADF-STEM images of the Pd@Pt concave decahedra with 47.5 wt $\%$ Pt taken at different tilting angles: (a) 60, (b) 35, (c) 15, (d) 0, (e) -15 , (f) -25 , (g) -40 , and (h) -55 degrees. 


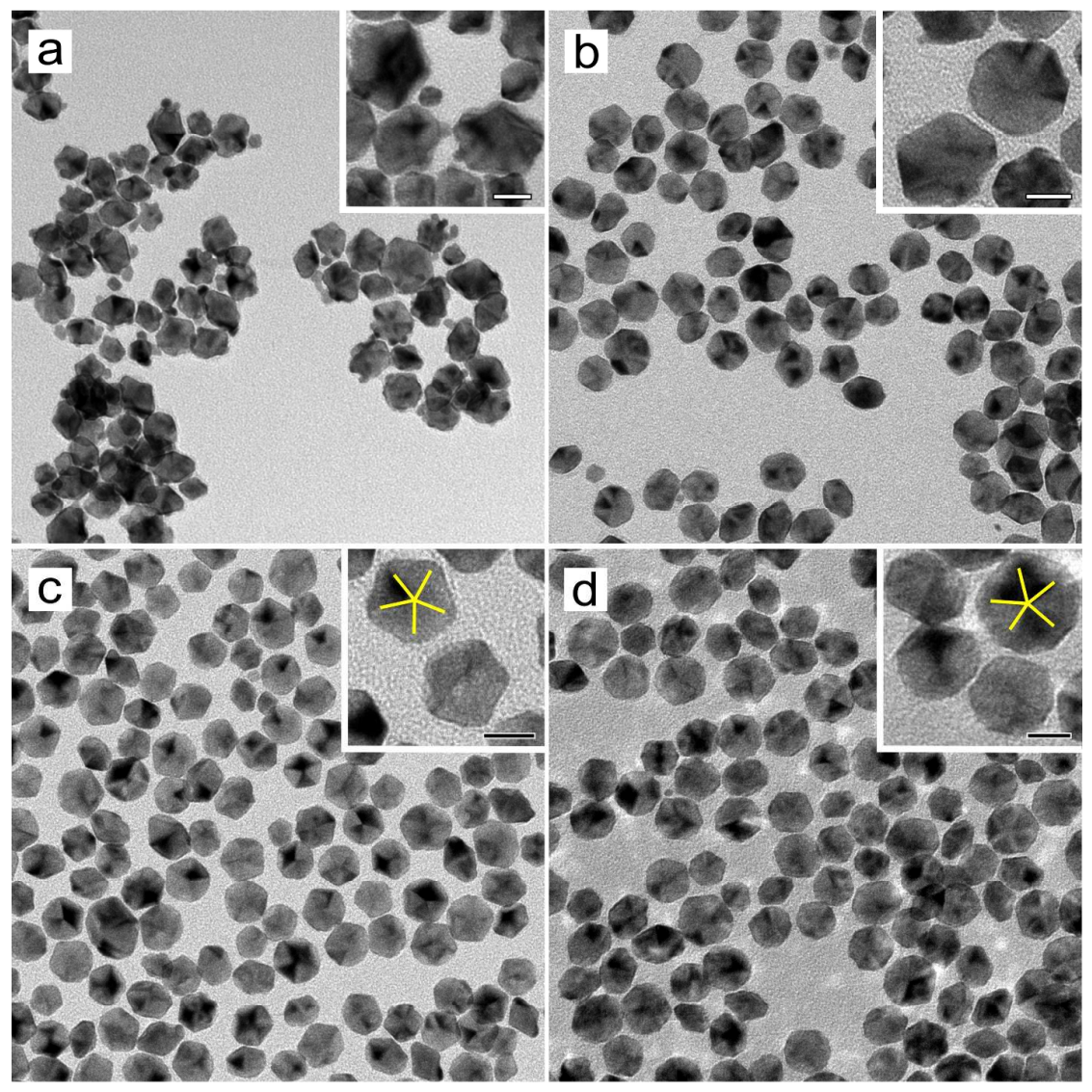

$-20 \mathrm{~nm}$

Figure S5. (a) TEM image of nanocrystals synthesized using the standard procedure for the concave decahedra with $47.5 \mathrm{wt} \% \mathrm{Pt}$, except for the use of a faster injection rate $(4 \mathrm{~mL} / \mathrm{h} v s$. $1.5 \mathrm{~mL} / \mathrm{h}$ ) for the Pt precursor solution. The product contained both concave decahedra and multipods. (b) TEM image of the Pd@Pt concave decahedra with 47.5 wt\% Pt, which was prepared using the standard protocol. (c, d) TEM images of nanocrystals prepared using the standard protocol for the concave decahedra with $47.5 \mathrm{wt} \% \mathrm{Pt}$, except that the reaction temperature was decreased from $200{ }^{\circ} \mathrm{C}$ to (c) $140{ }^{\circ} \mathrm{C}$ and (d) $110{ }^{\circ} \mathrm{C}$, respectively. The scale bars in the insets are $10 \mathrm{~nm}$. 

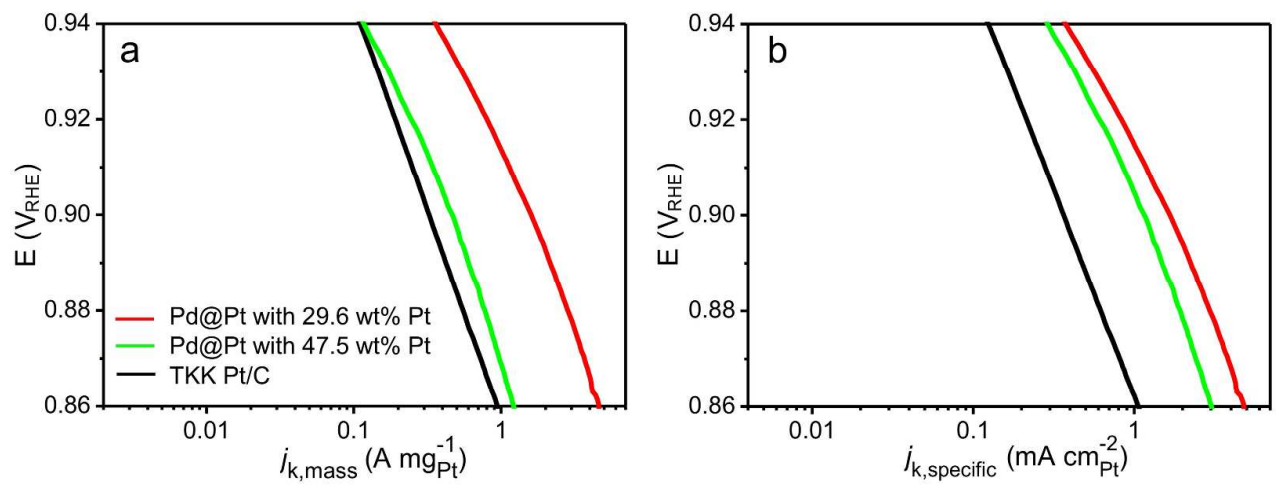

Figure S6. (a) Mass and (b) specific activities of the catalysts presented as the kinetic current density $\left(j_{\mathrm{k}}\right)$ normalized to the corresponding mass of Pt and ECSA, respectively. 


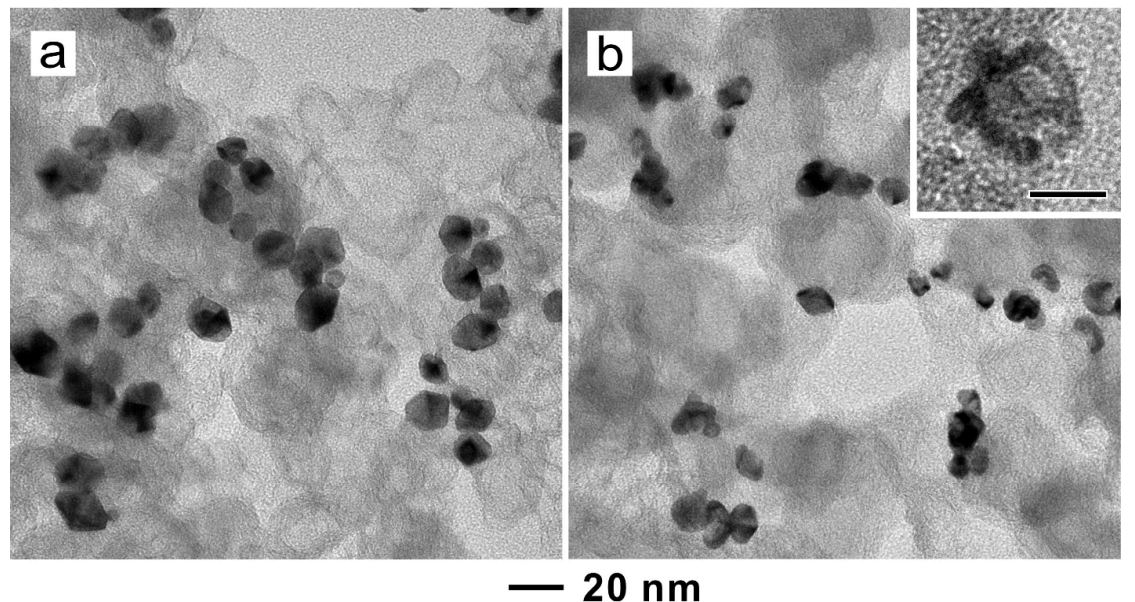

Figure S7. TEM images of the carbon-supported Pd@Pt concave decahedra with 29.6 wt $\%$ Pt (a) before and (b) after 10,000 cycles of the accelerated durability test. The scale bar in the inset of (b) is $10 \mathrm{~nm}$. 
Table S1. Calculated angles between high-index $\{h k k\}$ facets and $\{100\}$ edge facets.

\begin{tabular}{ccc}
\hline$h$ & $k$ & Angle with $\{100\}$ \\
\hline 9 & 1 & $8.93^{\circ}$ \\
10 & 1 & $8.05^{\circ}$ \\
15 & 1 & $5.39^{\circ}$ \\
16 & 1 & $5.05^{\circ}$ \\
20 & 1 & $4.04^{\circ}$ \\
\hline
\end{tabular}


Table S2. Specific ECSAs of the Pt/C catalyst from TKK and two other catalysts based on the Pd@Pt concave decahedra.

\begin{tabular}{cccc}
\hline & TKK Pt/C & $\begin{array}{l}\text { Pd@Pt with } \\
29.6 \mathrm{wt} \% \mathrm{Pt}\end{array}$ & $\begin{array}{c}\text { Pd@Pt with } \\
47.5 \mathrm{wt} \% \mathrm{Pt}\end{array}$ \\
\hline $\begin{array}{c}\text { Specific } \\
\text { ECSA }\left(\mathrm{m}^{2} \mathrm{~g}^{-1}\right)\end{array}$ & 88.9 & 95.9 & 59.4 \\
\hline
\end{tabular}


Supporting Movie Legend

Movie S1. Video of HAADF-STEM images taken at different tilt angles for the Pd@Pt concave decahedra with $47.5 \mathrm{wt} \% \mathrm{Pt}$. 